\title{
Boundary feedback control of 2-D shallow water equations
}

\author{
Ben Mansour Dia · Jesper Oppelstrup
}

Received: 19 December 2012 / Revised: 19 February 2013 / Accepted: 19 February 2013 / Published online: 13 March 2013

(C) Springer-Verlag Berlin Heidelberg 2013

\begin{abstract}
Local explicit feedback boundary conditions are given for the stabilization in $L^{2}$-norm of the 2-D shallow water model. The proposed method is based on symmetrization of the flux matrices of the linearized model and analysis of the Riemann invariants. The non-conservative 2-D shallow water equations are linearized around the target steady state sub-critical flow. The established feedback control laws guarantee a decay of the energy of the perturbation model. We present numerical simulations to demonstrate how the proposed controller works for the linearized as well as nonlinear shallow water problem.
\end{abstract}

Keywords 2-D shallow water equations .

Water management $\cdot$ Boundary control $\cdot$ Symmetrization

\section{Mathematics Subject Classification (2000) 35L50 .} 76B75 $\cdot 93 \mathrm{D} 15 \cdot 93 \mathrm{~B} 52$

\section{Introduction}

The fundamentals of control theory for partial differential equations (PDE) and their ramifications were established by J. L. Lions and Russell in [12] and [13] respectively. Water

This work is supported by grants from ISP (International Science Programme) of Uppsala university.

B. M. Dia $(\varangle)$

UFR de Sciences Appliquées et Technologies, Université Gaston

Berger, BP 234, Saint-Louis, Sénégal

e-mail: mansourben2002@yahoo.fr

J. Oppelstrup

NA-gruppen, CSC, Royal Institute of Technology,

10044 Stockholm, Sweden

e-mail: jespero@nada.kth.se flow regulation problems are of considerable societal interest, and we consider here the application to river engineering. Among the several approaches to design a control law, we mention the Lyapunow method, the Linear-Quadratic control method, Robust $H_{\infty}$ control, and Boundary PI regulation, etc. The technique of LQ control for water level regulation is proposed in [9]. This method is based on a linear symmetric hyperbolic infinite-dimensional form. In [14] it is presented a method based on robust fractional order controllers to regulate irrigation channels with variable dynamical parameters. In [3-5,7], authors developed control laws for 1-D shallow water (SW) equations by acting on the flow through the upstream and/or downstream boundaries.

In river and estuarine systems, control actions are usually implemented at either external or internal system boundaries. The well-posedness of overall system is determined by the relationship between the system state and control actions. While the characterization of the effect of each of the control actions on the system is still a challenge, advances have been made on the 2-D channel flow problem. For instance, a stabilization method for the parabolic equilibrium velocity profile in a $2-\mathrm{D}$ channel flow is proposed in $[1,2]$. The authors derived feedback laws based on linear optimal control which ensure global stability in $\mathrm{H}^{2}$-norm for the 2-D Navier Stokes equations. For free surface flow regulation problem, [6] shows how to build a local stabilizing boundary controller for the 2-D SW equations using 1-D exponential stability results for 1-D hyperbolic system. The established control law guarantees exponential decay of an approximation of the perturbation energy around a given steady state with a stabilization rate which can be selected to achieve some compromise between quick stabilization and control effort / cost.

In this paper, we address the problem of boundary control of 2-D SW equations. The control laws are derived from a 
linearization of the non-conservative form of the model around the desired steady state. The controller building process is based on a reformulation of the problem as a symmetric hyperbolic linear system. The control laws are defined through characteristic variables describing waves motions. The regulation task is to change the flow parameters to the sub-critical set point. The control laws are defined at each point of the controlled part of the boundary by acting on the incoming gravity waves and the developed boundary conditions imply stability in $L^{2}$-norm of the perturbation state. A high resolution finite volume method is used to apply the designed feedback law to the conservative nonlinear 2-D SW equations to illustrate the performance of the controller. The algorithm is an extension of the 1-D wave propagation approach that captures the cross-derivative terms needed for second order accuracy while allowing the use of simple 1$\mathrm{D}$ limiters. The numerical results carry the message that the proposed controllers work for the 2-D nonlinear stabilization problem, albeit with perturbation energy which does not tend monotonically to zero.

The organization of this paper is as follows. In sect. 2 we present the SW equations in the non-conservative form, their linearization and we state the linear stabilization problem and the main result. Section 3 is devoted to algebraic computations in order to rewrite the linear model as an hyperbolic linear system with symmetric flux matrices. In this section, we define also physical variables describing waves motion. Section 4 deals with justification of setting boundary conditions of the control problem by establishing the admissible ones. Section 5 gives an overview of the proof of the main result, that is to say, energy estimation, boundary control design and existence of solution of the control problem. In sect. 6, we present numerical experiments for the linearized as well as nonlinear model and investigate the effect of varying the actuators for the feedback laws.

\section{Problem statement, main result}

The SW equations are a system of hyperbolic PDE. They are derived from the principles of conservation of mass and conservation of momentum. Under the shallowness condition, they are derived from depth-integrating the Navier Stokes equations. In the case of no Coriolis effect, frictional and diffusion effect, the 2-D SW in the non-conservative form are :

$$
\begin{cases}\partial_{t} h+\partial_{x}(h u)+\partial_{y}(h v)=0 & \text { in } \mathcal{Q}, \\ \partial_{t} u+g \partial_{x} h+u \partial_{x} u+v \partial_{y} u=0 & \text { in } \mathcal{Q}, \\ \partial_{t} v+g \partial_{y} h+u \partial_{x} v+v \partial_{y} v=0 & \text { in } \mathcal{Q}, \\ \text { boundary and initial conditions, } & \end{cases}
$$

where $(t,(x, y)) \in \mathcal{Q}=(0, T) \times \Omega$, the real $T$ is a positive number. The domain $\Omega$ is a fixed bounded open subset of $\mathbb{R}^{2}$. The variable $h$ designates the height of the water column, $(u, v)$ the 2 -D velocity field on the reference $(O x, O y)$. The gravitational force acting on the fluid is represented by the constant $g$. The symbol $\partial_{t}$ designates the time derivative while $\partial_{x}$ and $\partial_{y}$ are the space derivatives in the $x$ - and $y$-directions respectively.

\subsection{Linearization}

Let us recall that we are looking for suitable boundary conditions to bring the flow variables to the equilibrium set $(\bar{h}, \bar{u}, \bar{v})$ which is solution of the stationary problem associated to (1). Such boundary conditions can be performed using a linear model governing the perturbation state around the constant steady state $(\bar{h}, \bar{u}, \bar{v})$. The perturbation state is denoted by $(\tilde{h}, \tilde{u}, \tilde{v})$ and it is introduced as the difference between the present state $(h, u, v)$ and the set point $(\bar{h}, \bar{u}, \bar{v})$

$$
(\tilde{h}, \tilde{u}, \tilde{v})(t, x, y)=(h, u, v)(t, x, y)-(\bar{h}, \bar{u}, \bar{v}) .
$$

We plug (2) into (1) and assume $|\tilde{h}| \ll \bar{h},|\tilde{u}| \ll|\bar{u}|$ and $|\tilde{v}| \ll|\bar{v}|$, so we can neglect the nonlinear perturbation terms to write the following linearized model :

$$
\left\{\begin{array}{l}
\partial_{t} \tilde{U}+A \partial_{x} \tilde{U}+B \partial_{y} \tilde{U}=0 \text { in } \mathcal{Q}, \\
\text { boundary and initial conditions }
\end{array}\right.
$$

where $\tilde{U}$ is the residual state vector, $A$ and $B$ are the flux matrices

$$
\tilde{U}=\left(\begin{array}{c}
\tilde{h} \\
\tilde{u} \\
\tilde{v}
\end{array}\right), \quad A=\left(\begin{array}{ccc}
\bar{u} & \bar{h} & 0 \\
g & \bar{u} & 0 \\
0 & 0 & \bar{u}
\end{array}\right) \text { and } B=\left(\begin{array}{ccc}
\bar{v} & 0 & \bar{h} \\
0 & \bar{v} & 0 \\
g & 0 & \bar{v}
\end{array}\right) \text {. }
$$

\subsection{Notations}

The boundary of the 2-D domain $\Omega$ is denoted by $\partial \Omega$. The vector $\mathbf{n}=\left(n_{x}, n_{y}\right)^{t r}$ is the normal unit external vector at the boundary, the tangential unit vector is then $\boldsymbol{\tau}=\left(-n_{y}, n_{x}\right)^{t r}$. The quantity $\mathbf{u}_{n}=\bar{u} n_{x}+\bar{v} n_{y}$ is standing for the normal steady velocity. The flow is supposed sub-critical so that the equilibrium parameters $\bar{h}, \bar{u}$ and $\bar{v}$ are non-null and the Froude number $F$ satisfies

$F=\frac{\|(\bar{u}, \bar{v})\|_{2}}{\sqrt{g \bar{h}}}<1$.

For the stabilization purpose, we adopt also the assumption that the boundary $\partial \Omega$ is separated into two disjoint subsets $\Gamma_{n c}$ and $\Gamma_{c}$ defined by

$$
\Gamma_{n c}=\left\{(x, y) \in \partial \Omega: \mathbf{u}_{n} \geq 0\right\} \text { and } \Gamma_{c}=\Gamma_{c x} \cup \Gamma_{c y}
$$




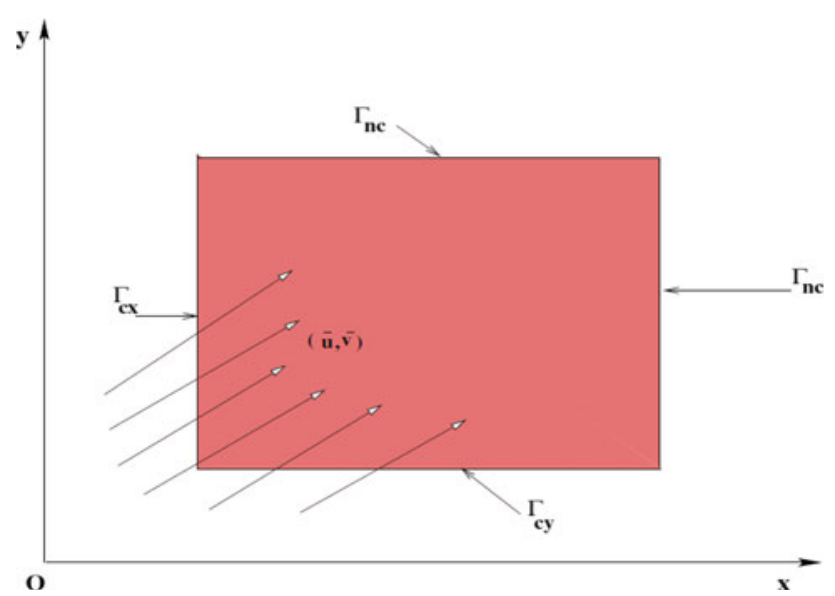

Fig. 1 Geometry of a controlled domain

where

$\Gamma_{c x}=\left\{(x, y) \in \partial \Omega: \bar{u} n_{x} \leq 0\right.$ and $\left.n_{y}=0\right\}$,

$\Gamma_{c y}=\left\{(x, y) \in \partial \Omega: \bar{v} n_{y} \leq 0\right.$ and $\left.n_{x}=0\right\}$.

The subset $\Gamma_{c}$ is the controllable portion of the boundary while $\Gamma_{n c}$ denotes the uncontrollable part. Geometries satisfying those assumptions are easy to construct. The next figure (Fig. 1) illustrates the frontier subsets design with respect to the perturbation flow direction on a rectangular domain.

\subsection{Stabilization problem}

We consider the following stabilization problem by boundary control

$$
\begin{cases}\partial_{t} \tilde{U}+A \partial_{x} \tilde{U}+B \partial_{y} \tilde{U}=0 & \text { in } \mathcal{Q}, \\ \tilde{U}(t=0, x, y)=\tilde{U}^{0}(x, y) & \text { in } \Omega, \\ \tilde{u} n_{x}+\tilde{v} n_{y}=0 & \text { on }(0,+\infty) \times \Gamma_{n c}, \\ (\tilde{u}, \tilde{v})=\left(\mathcal{V}_{1}, 0\right) & \text { on }(0,+\infty) \times \Gamma_{c x}, \\ (\tilde{u}, \tilde{v})=\left(0, \mathcal{V}_{2}\right) & \text { on }(0,+\infty) \times \Gamma_{c y} .\end{cases}
$$

In the initial-boundary value problem (5), the boundary velocity vector $\left(\mathcal{V}_{1}, 0\right)$ on $\Gamma_{c x}$ and $\left(0, \mathcal{V}_{2}\right)$ on $\Gamma_{c y}$ shall be chosen such that the perturbation $(\tilde{h}, \tilde{u}, \tilde{v})$ vanishes.

Definition 1 The energy $E$ of (5) is defined as follows

$E(t)=\int_{\Omega}\left(\tilde{h}^{2}+\frac{\bar{h}}{g} \tilde{u}^{2}+\frac{\bar{h}}{g} \tilde{v}^{2}\right) d \Omega$.

The quantity to be stabilized is the energy $E(t)$, a measurement of the variables $\tilde{h}, \tilde{u}$ and $\tilde{v}$. The energy $E(t)$ is equivalent to the $L^{2}$-norm on $\Omega$ of the vector field $(\tilde{h}, \tilde{u}, \tilde{v})$. It vanishes only if the perturbation state vanishes over all the domain $\Omega$. Typically, $E$ is a type of weakened Lyapunov function candidate since vanishing of variables $(\tilde{h}=\tilde{u}=$ $\tilde{v}=0$ ) at some time $t$ at the boundary do not ensure $E=0$. The boundary control $\mathcal{V}_{1}$ and $\mathcal{V}_{2}$ will be established such that $E$ decreases with increasing time.

\subsection{Function space}

Let us recall that $T$ is a positive number and $\mathcal{Q}=(0, T) \times \Omega$, we define the differential operator $D i v$ for the vector function $f=\left(f_{1}, f_{2}, f_{3}\right)^{T}$ by

$\operatorname{Divf}=\partial_{t} f_{1}+\partial_{x} f_{2}+\partial_{y} f_{3}$.

In this sense, we set the function space $H(\operatorname{Div}, \mathcal{Q})$ by

$H(\operatorname{Div}, \mathcal{Q})=\left\{\Psi \in L^{2}(\mathcal{Q})^{3} ; \quad \operatorname{Div}(\Psi) \in L^{2}(\mathcal{Q})\right\}$.

If the vector $(\tilde{h}, \tilde{u}, \tilde{v})$ the solution of the system (5) is in $L^{2}(\mathcal{Q})^{3}$, then for $i=1,2,3 X_{i} \in H(\operatorname{Div}, \mathcal{Q})$ since $\operatorname{Div}\left(X_{i}\right)=0 \in L^{2}(\mathcal{Q})$ where

$$
\begin{aligned}
& X_{1}(\tilde{h}, \tilde{u}, \tilde{v})=\left(\begin{array}{l}
\tilde{h} \\
\bar{u} \tilde{h}+\bar{h} \tilde{u} \\
\bar{v} \tilde{h}+\bar{h} \tilde{v}
\end{array}\right), \quad X_{2}(\tilde{h}, \tilde{u}, \tilde{v})=\left(\begin{array}{l}
\tilde{u} \\
g \tilde{h}+\bar{u} \tilde{u} \\
\bar{v} \tilde{u}
\end{array}\right) \\
& \text { and } X_{3}(\tilde{h}, \tilde{u}, \tilde{v})=\left(\begin{array}{l}
\tilde{v} \\
\bar{u} \tilde{v} \\
g \tilde{h}+\bar{v} \tilde{v}
\end{array}\right) .
\end{aligned}
$$

In this sense, we define the functional space $V$ by

$$
\begin{aligned}
& V=\left\{\begin{array}{l}
(\tilde{h}, \tilde{u}, \tilde{v}) \in L^{\infty}\left(0, T ; L^{2}(\Omega)^{3}\right): \quad \text { for } i=1,2,3 \\
X_{i}(\tilde{h}, \tilde{u}, \tilde{v}) \in H(\operatorname{Div}, \mathcal{Q}),
\end{array}\right. \\
& \left.\quad(\tilde{u}, \tilde{v})_{\mid \Gamma_{n c}} \cdot \mathbf{n}=0, \quad \tilde{v}_{\mid \Gamma_{c x}}=0 \text { and } \tilde{u}_{\mid \Gamma_{c y}}=0\right\} .
\end{aligned}
$$

The trace of the solution $(\tilde{h}, \tilde{u}, \tilde{v})$ belonging to $V$ makes sense in $H^{-\frac{1}{2}}(\partial \mathcal{Q})$ (see [8], p. 27).

\subsection{Main result}

The boundary conditions $\left(\mathcal{V}_{1}, 0\right)$ and $\left(0, \mathcal{V}_{2}\right)$ for the stabilization problem (5) will be sought in such a way to decrease the energy $E$ in time. Those boundary conditions are built by using dimensional splitting Riemann invariants analysis. The next theorem gives the existence of such boundary conditions.

Theorem 1 If the initial conditions $\tilde{U}^{0} \in L^{2}(\Omega)^{3}$, then there exists a feedback boundary controller $\left(\mathcal{V}_{1}, 0\right)$ on $\Gamma_{c x}$ and $\left(0, \mathcal{V}_{2}\right)$ on $\Gamma_{c y}$ such that the control system (5) has an unique solution $\tilde{U} \in V$ which is stable around $(0,0,0)^{T}$ in the sense that

$E(t) \leq C_{0} E(0)$ with $C_{0}>0$

This theorem is the main result of this paper and it is proved in sect. 5. In fact, we shall prove the existence of the solution and we shall build boundary controllers $\mathcal{V}_{1}$ and $\mathcal{V}_{2}$ to make time derivative of $E$ non-positive.

To say the system (5) is stable means that the solution $\tilde{U}$ does not grow. Further refinement of the result is required to 
establish e.g. exponential asymptotic stability which would guarantee the decay to zero of the energy when time tends to infinity. One may conjecture that since for sub-critical flows, all three wave-speeds are non-vanishing everywhere, and so all perturbations will propagate to the boundary where some of their energy is radiated. But, in contrast to the system (5), they also reflect so we do not expect the perturbations to vanish in finite time.

\section{Eigenstructure}

\subsection{Symmetrization}

The symmetrization proceeds by introducing new variables in order to get a hyperbolic system with symmetric matrices. Multiplying the hyperbolic system of equations $\partial_{t} \tilde{U}+$ $A \partial_{x} \tilde{U}+B \partial_{y} \tilde{U}=0$ by the diagonal matrix $D=$ $\operatorname{diag}\{1, \sqrt{\bar{h} / g}, \sqrt{\bar{h} / g}\}$, we obtain

$$
\partial_{t} D \tilde{U}+D A \partial_{x} \tilde{U}+D B \partial_{y} \tilde{U}=0
$$

which can be written as follows

$\partial_{t} D \tilde{U}+D A D^{-1} D \partial_{x} \tilde{U}+D B D^{-1} D \partial_{y} \tilde{U}=0$.

Denoting variables vector $\hat{U}=D \tilde{U}$ and matrices $\hat{A}=$ $D A D^{-1}$ and $\hat{B}=D B D^{-1}$, it comes the following hyperbolic system

$\partial_{t} \hat{U}+\hat{A} \partial_{x} \hat{U}+\hat{B} \partial_{y} \hat{U}=0$

where the flux matrices $\hat{A}$ and $\hat{B}$ are defined as

$$
\hat{A}=\left(\begin{array}{ccc}
\bar{u} & c & 0 \\
c & \bar{u} & 0 \\
0 & 0 & \bar{u}
\end{array}\right) \quad \text { and } \quad \hat{B}=\left(\begin{array}{ccc}
\bar{v} & 0 & c \\
0 & \bar{v} & 0 \\
c & 0 & \bar{v}
\end{array}\right) \text {, }
$$

the quantity $c=\sqrt{g \bar{h}}$ is the wave speed.

\subsection{Characteristic variables}

Using characteristic variables analysis is a practical means to ensure well-posedness of hyperbolic initial-boundary value problems. In this part, we write out the characteristic variables related to $x$-direction and $y$-direction. Thus, we denote $\lambda_{1}^{a}, \lambda_{2}^{a}, \lambda_{3}^{a}$ the 3 eigenvalues of the matrix $\hat{A}$ and $\lambda_{1}^{b}, \lambda_{2}^{b}, \lambda_{3}^{b}$ the 3 eigenvalues of the matrix $\hat{B}$ :

$$
\begin{aligned}
& \lambda_{1}^{a}=\bar{u}-c, \quad \lambda_{2}^{a}=\bar{u}+c, \quad \lambda_{3}^{a}=\bar{u} \quad \text { and } \lambda_{1}^{b}=\bar{v}-c, \\
& \quad \lambda_{2}^{b}=\bar{v}+c, \quad \lambda_{3}^{b}=\bar{v} .
\end{aligned}
$$

The quantities $\lambda_{1}^{a}$ and $\lambda_{2}^{a}$ (resp. $\lambda_{1}^{b}$ and $\lambda_{2}^{b}$ ) are of constant opposite sign, they are gravity velocities and their signs do not depend on the steady state while the $\operatorname{sign}$ of $\lambda_{3}^{a}\left(\operatorname{resp} . \lambda_{3}^{b}\right)$ is determined by the equilibrium flow direction. We denote by
$P$ and $Q$ the transformation matrices of $\hat{A}$ and $\hat{B}$ respectively, defined as follow

$$
P=\left(\begin{array}{lll}
1 & 1 & 0 \\
-1 & 1 & 0 \\
0 & 0 & \sqrt{2}
\end{array}\right), \quad Q=\left(\begin{array}{lll}
1 & 1 & 0 \\
0 & 0 & \sqrt{2} \\
-1 & 1 & 0
\end{array}\right)
$$

The diagonal matrices $\Lambda_{a}$ and $\Lambda_{b}$ are the matrices of eigenvalues of $\hat{A}$ and $\hat{B}$ respectively, written in the following form

$$
\Lambda_{a}=\operatorname{diag}\left\{\lambda_{1}^{a}, \lambda_{2}^{a}, \lambda_{3}^{a}\right\} \text { and } \Lambda_{b}=\operatorname{diag}\left\{\lambda_{1}^{b}, \lambda_{2}^{b}, \lambda_{3}^{b}\right\} .
$$

Since the matrices $\hat{A}$ and $\hat{B}$ are symmetric, the transformation matrices of eigenvectors $P$ and $Q$ are orthogonal. Therefore it comes that

$$
\begin{aligned}
& \hat{A}=\frac{1}{2} P \Lambda_{a} P^{-1}=\frac{1}{2} P \Lambda_{a} P^{t r} \\
& \text { and } \hat{B}=\frac{1}{2} Q \Lambda_{b} Q^{-1}=\frac{1}{2} Q \Lambda_{b} Q^{t r} .
\end{aligned}
$$

Let us denote

$$
\begin{aligned}
& \xi_{a}=\left(\xi_{a_{1}}, \xi_{a_{2}}, \xi_{a_{3}}\right)^{t r}, \quad \xi_{b}=\left(\xi_{b_{1}}, \xi_{b_{2}}, \xi_{b_{3}}\right)^{t r} \\
& \xi_{a}=P^{t r} \hat{U}, \quad \xi_{b}=Q^{t r} \hat{U}
\end{aligned}
$$

where

$$
\begin{aligned}
\xi_{a_{1}}(t, x, y)= & \tilde{h}(t, x, y)-\sqrt{(\bar{h} / g)} \tilde{u}(t, x, y), \\
& \xi_{b_{1}}(t, x, y)=\tilde{h}(t, x, y)-\sqrt{(\bar{h} / g)} \tilde{v}(t, x, y), \\
\xi_{a_{2}}(t, x, y)= & \tilde{h}(t, x, y)+\sqrt{(\bar{h} / g)} \tilde{u}(t, x, y), \\
& \xi_{b_{2}}(t, x, y)=\tilde{h}(t, x, y)+\sqrt{(\bar{h} / g)} \tilde{v}(t, x, y), \\
\xi_{a_{3}}(t, x, y)= & \sqrt{(2 \bar{h} / g)} \tilde{v}(t, x, y), \\
& \xi_{b_{3}}(t, x, y)=\sqrt{(2 \bar{h} / g)} \tilde{u}(t, x, y)
\end{aligned}
$$

and we state the following definition.

Definition 2 The variables $\xi_{a_{i}}$ (resp. $\xi_{b_{i}}$ ) for $i=1,2,3$ are called $x$-dimensional splitting characteristic variables ( $x$-DSCV) (resp. $y$-dimensional splitting characteristic variables $(y$-DSCV $))$.

Physically $\xi_{a_{i}}$ and $\xi_{b_{i}}(\mathrm{i}=1,2,3)$ are viewed as characteristic variables respectively on $x$ and $y$ direction in the dimensional splitting framework. The variables $\xi_{a_{1,2}}$ and $\xi_{b_{1,2}}$ are the gravity waves, on which one acts in order to change the state of the flow towards the steady state. Thus, the prospective controllers $\mathcal{V}_{1}$ and $\mathcal{V}_{2}$ will be built by acting on those waves. The variable $\xi_{a_{3}}$ is the wave orthogonal to the splitting $x$-direction. Acting on it in the $x$-direction provides little effect and creates well-posedness concerns with respect to the action defined on $\xi_{b_{1,2}}$. However, $\xi_{a_{3}}$ and $\xi_{b_{3}}$ will be affected respectively by the actions defined on $\xi_{b_{1,2}}$ and $\xi_{a_{1,2}}$. 


\section{Admissible boundary conditions}

The setting class of boundary conditions is delineated by well-posedness requirements. This is important in the sense that the boundary conditions should ensure the wellposedness of the associated Cauchy problem. Boundary conditions which ensure well-posedness of the initial-boundary value problem are called admissible. For simple 1-D hyperbolic initial-boundary value problem $\partial_{t} w+\gamma \partial_{x} w=0$ for $(t, x) \in[0, T] \times[0, L]$, the boundary control law is defined by acting on the incoming wave in the domain $[0, L]$. This is related to the sign of the wave speed $\gamma$. This analysis serves as basis for proceeding to a priori estimation to establish admissible boundary conditions on $\Gamma_{c}$ and on $\Gamma_{n c}$.

We are looking for boundary conditions for a system of 3 equations with 3 unknowns. To establish admissible boundary conditions for the control problem (5), we process to a weak formulation of the hyperbolic system with symmetric flux matrices without worrying about regularity aspects. Let $\psi=\left(\varphi, w_{1}, w_{2}\right)^{t r}$ be a smooth function, we integrate the product of $\psi^{t r}$ by $\partial_{t} \hat{U}+\hat{A} \partial_{x} \hat{U}+\hat{B} \partial_{y} \hat{U}=0$ to write

$$
\begin{aligned}
& \int_{\Omega} \psi^{t r} \partial_{t} \hat{U} d \Omega-\frac{1}{2} \int_{\Omega}\left(\partial_{x} \Phi_{a}^{t r}\right) \Lambda_{a} \xi_{a} d \Omega \\
& -\frac{1}{2} \int_{\Omega}\left(\partial_{y} \Phi_{b}^{t r}\right) \Lambda_{b} \xi_{b} d \Omega \\
& =-\frac{1}{2} \int_{\partial \Omega} \Phi_{a}^{t r} \Lambda_{a} \xi_{a} n_{x} d \sigma-\frac{1}{2} \int_{\partial \Omega} \Phi_{b}^{t r} \Lambda_{b} \xi_{b} n_{y} d \sigma,
\end{aligned}
$$

where $\Phi_{a}=P^{t r} \psi$ and $\Phi_{b}=Q^{t r} \psi$. The idea behind this a priori estimation is to fix the sign of each RHS term according to the hypothesis of our control problem (5) when test function $\psi$ is replaced by the state $\hat{U}$. The boundary conditions must be chosen on those terms having unknown sign. The RHS of (11) can be split into boundary terms on $\Gamma_{c}$ and boundary terms on $\Gamma_{n c}$ denoted by $T_{c}$ and $T_{n c}$ respectively

$$
\begin{gathered}
\int_{\Omega} \psi^{t r} \partial_{t} \hat{U} d \Omega-\frac{1}{2} \int_{\Omega}\left(\partial_{x} \Phi_{a}^{t r}\right) \Lambda_{a} \xi_{a} d \Omega \\
-\frac{1}{2} \int_{\Omega}\left(\partial_{y} \Phi_{b}^{t r}\right) \Lambda_{b} \xi_{b} d \Omega=T_{c}+T_{n c}
\end{gathered}
$$

where

$$
\begin{aligned}
& T_{c}=-\frac{1}{2} \int_{\Gamma_{c}}\left(\Phi_{a}^{t r} \Lambda_{a} \xi_{a} n_{x}+\Phi_{b}^{t r} \Lambda_{b} \xi_{b} n_{y}\right) d \sigma, \\
& T_{n c}=-\frac{1}{2} \int_{\Gamma_{n c}}\left(\Phi_{a}^{t r} \Lambda_{a} \xi_{a} n_{x}+\Phi_{b}^{t r} \Lambda_{b} \xi_{b} n_{y}\right) d \sigma .
\end{aligned}
$$

On the uncontrollable boundary portion $\Gamma_{n c}$, we have

$$
\begin{aligned}
T_{n c}= & -\frac{1}{2} \int_{\Gamma_{n c}}\left(\lambda_{1}^{a} \Phi_{a_{1}} \xi_{a_{1}}+\lambda_{2}^{a} \Phi_{a_{2}} \xi_{a_{2}}+\lambda_{3}^{a} \Phi_{a_{2}} \xi_{a_{3}}\right) n_{x} d \sigma \\
& -\frac{1}{2} \int_{\Gamma_{n c}}\left(\lambda_{1}^{b} \Phi_{b_{1}} \xi_{b_{1}}+\lambda_{2}^{b} \Phi_{b_{2}} \xi_{b_{2}}+\lambda_{3}^{b} \Phi_{b_{2}} \xi_{b_{3}}\right) n_{y} d \sigma \\
= & -\int_{\Gamma_{n c}}\left(\tilde{h} \varphi+(\bar{h} / g) w_{1} \tilde{u}+(\bar{h} / g) w_{2} \tilde{v}\right)(\bar{u}, \bar{v}) \cdot \mathbf{n} d \sigma \\
& -\bar{h} \int_{\Gamma_{n c}} \varphi\left(\tilde{u} n_{x}+\tilde{v} n_{y}\right) d \sigma-\bar{h} \int_{\Gamma_{n c}} \tilde{h}\left(w_{1} n_{x}+w_{2} n_{y}\right) d \sigma .
\end{aligned}
$$

Since the normal steady flow $(\bar{u}, \bar{v}) \cdot \mathbf{n}$ is positive on $\Gamma_{n c}$, it comes from (12) that the boundary conditions in this part should be given on $\tilde{u} n_{x}+\tilde{v} n_{y}$ or $\tilde{h}$.

On the controllable boundary part $\Gamma_{c}$, we have

$$
\begin{aligned}
T_{c}= & -\frac{1}{2} \int_{\Gamma_{c}}\left(\lambda_{1}^{a} \Phi_{a_{1}} \xi_{a_{1}}+\lambda_{2}^{a} \Phi_{a_{2}} \xi_{a_{2}}+\lambda_{3}^{a} \Phi_{a_{2}} \xi_{a_{3}}\right) n_{x} d \sigma \\
& -\frac{1}{2} \int_{\Gamma_{c}}\left(\lambda_{1}^{b} \Phi_{b_{1}} \xi_{b_{1}}+\lambda_{2}^{b} \Phi_{b_{2}} \xi_{b_{2}}+\lambda_{3}^{b} \Phi_{b_{2}} \xi_{b_{3}}\right) n_{y} d \sigma \\
= & -\frac{1}{2} \int_{\Gamma_{c x}}\left(\Phi_{a_{1}} \xi_{a_{1}}+\Phi_{a_{2}} \xi_{a_{2}}+\Phi_{a_{2}} \xi_{a_{3}}\right) \bar{u} n_{x} d \sigma \\
& -\frac{c}{2} \int_{\Gamma_{c x}}\left(-\Phi_{a_{1}} \xi_{a_{1}}+\Phi_{a_{2}} \xi_{a_{2}}\right) n_{x} d \sigma \\
& -\frac{1}{2} \int_{\Gamma_{c y}}\left(\Phi_{b_{1}} \xi_{b_{1}}+\Phi_{b_{2}} \xi_{b_{2}}+\Phi_{b_{2}} \xi_{b_{3}}\right) \bar{v} n_{y} d \sigma \\
& -\frac{c}{2} \int_{\Gamma_{c y}}\left(-\Phi_{b_{1}} \xi_{b_{1}}+\Phi_{b_{2}} \xi_{b_{2}}\right) n_{y} d \sigma
\end{aligned}
$$

The quantities $\bar{u} n_{x}$ and $\bar{v} n_{y}$ are negative on $\Gamma_{c}$. Then the relation (13) indicates that control laws should be sought by acting on the waves $\xi_{a_{1,2}}$ on $\Gamma_{c x}$ and $\xi_{b_{1,2}}$ on $\Gamma_{c y}$. This is to say that the controllers should be chosen in the class

$$
(\tilde{u}, \tilde{v})(t, x, y)=K(t, x, y) \tilde{h}(t, x, y)\left(n_{x}, n_{y}\right)
$$

by proper choice of $K$ which is the starting point for the analysis by characteristics.

\section{Controller building process}

This section is devoted to the proof of the Theorem 1. Thus, we build the setting boundary conditions in the system (5) and prove the existence of solution $\tilde{U}$. Nevertheless, we start by estimating the defined energy $E(t)$ of the perturbation state. 


\subsection{Energy estimation}

We replace the test function $\psi$ by the state $\hat{U}$ in (11) to express the time derivative of the energy $E$ as follows

$$
\begin{aligned}
\frac{d E}{d t} & =-\frac{1}{2} \int_{\partial \Omega} \xi_{a}^{t r} \Lambda_{a} \xi_{a} n_{x} d \sigma-\frac{1}{2} \int_{\partial \Omega} \xi_{b}^{t r} \Lambda_{b} \xi_{b} n_{y} d \sigma \\
& =\frac{1}{2} R_{c}(t)+\frac{1}{2} R_{n c}(t),
\end{aligned}
$$

where

$R_{c}(t)=-\int_{\Gamma_{c}} \xi_{a}^{t r} \Lambda_{a} \xi_{a} n_{x} d \sigma-\int_{\Gamma_{c}} \xi_{b}^{t r} \Lambda_{b} \xi_{b} n_{y} d \sigma$

and

$R_{n c}(t)=-\int_{\Gamma_{n c}} \xi_{a}^{t r} \Lambda_{a} \xi_{a} n_{x} d \sigma-\int_{\Gamma_{n c}} \xi_{b}^{t r} \Lambda_{b} \xi_{b} n_{y} d \sigma$.

The quantity $R_{c}(t)$ regroups boundary terms on the controllable portion $\Gamma_{c}$ while $R_{n c}(t)$ stands for boundary terms on the uncontrollable part $\Gamma_{n c}$. We are expressing in details the quantities $R_{c}(t)$ and $R_{n c}(t)$. The idea is to figure out the sign of $R_{n c}(t)$ according to the hypothesis of our control problem and to write $R_{c}(t)$ in preparation for designing boundary controllers $\mathcal{V}_{1}$ and $\mathcal{V}_{2}$ to ensure the non-positivity of $d E(t) / d t$. The quantity $R_{n c}(t)$ is written in details as follows

$$
\begin{aligned}
R_{n c}(t)= & -2 \int_{\Gamma_{n c}}\left(\tilde{h}^{2}+(\bar{h} / g) \tilde{u}^{2}+(\bar{h} / g) \tilde{v}^{2}\right) \bar{u} n_{x} d \sigma \\
& -2 \int_{\Gamma_{n c}}\left(\tilde{h}^{2}+(\bar{h} / g) \tilde{v}^{2}+(\bar{h} / g) \tilde{u}^{2}\right) \bar{v} n_{y} d \sigma \\
& -4 \bar{h} \int_{\Gamma_{n c}} \tilde{h}\left(\tilde{u} n_{x}+\tilde{v} n_{y}\right) d \sigma .
\end{aligned}
$$

Taking account the boundary condition on $\Gamma_{n c}\left(\tilde{u} n_{x}+\tilde{v} n_{y}=\right.$ 0 ) of the system (5), it comes that

$$
R_{n c}(t)=-2 \int_{\Gamma_{n c}} \hat{U}^{t r} \hat{U} \mathbf{u}_{n} d \sigma
$$

Since the normal steady velocity $\mathbf{u}_{n}$ is non-negative on $\Gamma_{n c}$, so

$$
R_{n c}(t) \leq 0 \text { for } t>0 \text {. }
$$

The controllable portion $\Gamma_{c}=\Gamma_{c x} \cup \Gamma_{c y}$, consequently the quantity $R_{c}$ can be written as

$$
\begin{aligned}
R_{c}(t)= & -\lambda_{1}^{a} \int_{\Gamma_{c x}} \xi_{a_{1}}^{2} n_{x} d \sigma-\lambda_{2}^{a} \int_{\Gamma_{c x}} \xi_{a_{2}}^{2} n_{x} d \sigma-\lambda_{3}^{a} \int_{\Gamma_{c x}} \xi_{a_{3}}^{2} n_{x} d \sigma \\
& -\lambda_{1}^{b} \int_{\Gamma_{c y}} \xi_{b_{1}}^{2} n_{y} d \sigma-\lambda_{2}^{b} \int_{\Gamma_{c y}} \xi_{b_{2}}^{2} n_{y} d \sigma-\lambda_{3}^{b} \int_{\Gamma_{c y}} \xi_{b_{3}}^{2} n_{y} d \sigma .
\end{aligned}
$$

From the relation (14), the flow on the controllable portion should be normal to be admissible for the system (5), it comes that the tangential flow is null on this portion i.e $(\tilde{u}, \tilde{v})^{t r} \cdot \tau=$ 0 which implies that

$-\lambda_{3}^{a} \int_{\Gamma_{c x}} \xi_{a_{3}}^{2} n_{x} d \sigma-\lambda_{3}^{b} \int_{\Gamma_{c y}} \xi_{b_{3}}^{2} n_{y} d \sigma=0$

Therefore, the energy estimation (16) can be written in the following form

$$
\begin{aligned}
\frac{d E(t)}{d t}= & -\lambda_{1}^{a} \int_{\Gamma_{c x}} \xi_{a_{1}}^{2} n_{x} d \sigma-\lambda_{2}^{a} \int_{\Gamma_{c x}} \xi_{a_{2}}^{2} n_{x} d \sigma-\lambda_{1}^{b} \int_{\Gamma_{c y}} \xi_{b_{1}}^{2} n_{y} d \sigma \\
& -\lambda_{2}^{b} \int_{\Gamma_{c y}} \xi_{b_{2}}^{2} n_{y} d \sigma-\int_{\Gamma_{n c}} \hat{U}^{t r} \hat{U} \mathbf{u}_{n} d \sigma
\end{aligned}
$$

\subsection{Boundary control design: proof of the theorem 1}

Step 1 : Controllers design. We define the controllers by acting normally on the incoming gravity waves in the following way

$\xi_{a_{2}}(t, x, y)=\alpha(t) \xi_{a_{1}}(t, x, y)$ on $(0,+\infty) \times \Gamma_{c x}$,

$\xi_{b_{2}}(t, x, y)=\beta(t) \xi_{b_{1}}(t, x, y)$ on $(0,+\infty) \times \Gamma_{c y}$.

The time functions $\alpha$ and $\beta$ are the actuators and they are supposed to be different to 1 . Physically $\alpha=\beta=1$ corresponds to two gravity waves which neutralize themselves. The energy estimation (22) becomes

$$
\begin{aligned}
\frac{d E(t)}{d t}= & -\left(\frac{\lambda_{1}^{a}}{\lambda_{3}^{a}}+\frac{\lambda_{2}^{a}}{\lambda_{3}^{a}} \alpha^{2}(t)\right) \int_{\Gamma_{c x}} \xi_{a_{1}}^{2} \bar{u} n_{x} d \sigma \\
& -\left(\frac{\lambda_{1}^{b}}{\lambda_{3}^{b}}+\frac{\lambda_{2}^{b}}{\lambda_{3}^{b}} \beta^{2}(t)\right) \int_{\Gamma_{c y}} \xi_{b_{1}}^{2} \bar{v} n_{y} d \sigma \\
& -\int_{\Gamma_{n c}} \hat{U}^{t r} \hat{U} \mathbf{u}_{n} d \sigma .
\end{aligned}
$$


Finally, to render $d E(t) / d t$ non-positive, we choose $\alpha$ and $\beta$ such that

$$
\left\{\begin{array}{l}
\alpha, \beta \neq 1, \\
\frac{\lambda_{1}^{a}}{\lambda_{3}^{a}}+\frac{\lambda_{2}^{a}}{\lambda_{3}^{a}} \alpha^{2}(t) \leq 0 \\
\frac{\lambda_{1}^{b}}{\lambda_{3}^{b}}+\frac{\lambda_{2}^{b}}{\lambda_{3}^{b}} \beta^{2}(t) \leq 0
\end{array}\right.
$$

Such $\alpha$ and $\beta$ exist and guarantee the perturbation energy $E(t)$ decay in time.

From the control laws definition (23)-(24), we introduce the following velocity vector $\left(\mathcal{V}_{1}, 0\right)$ on $\Gamma_{c x}$ and $\left(0, \mathcal{V}_{2}\right)$ on $\Gamma_{c y}$ where

$$
\begin{aligned}
& \mathcal{V}_{1}(t, x, y)=\frac{\alpha(t)-1}{\alpha(t)+1} \sqrt{\frac{g}{\bar{h}}} \tilde{h}(t, x, y), \\
& \mathcal{V}_{2}(t, x, y)=\frac{\beta(t)-1}{\beta(t)+1} \sqrt{\frac{g}{\bar{h}}} \tilde{h}(t, x, y) .
\end{aligned}
$$

For $\alpha$ and $\beta$ satisfying (26), the feedback boundary controllers $\left(\mathcal{V}_{1}, 0\right)$ and $\left(0, \mathcal{V}_{2}\right)$ on $\Gamma_{c x}$ and $\Gamma_{c y}$ respectively guarantee a decay in time of the energy $E(t)$.

Step 2 : Existence of solution We prove here the existence of solution of the stabilization system (5) using the FaedoGalerkin method. Let us denote $\mathcal{U}_{a}=\left(\tilde{h}, \mathcal{V}_{1}, 0\right)^{t r}, \mathcal{U}_{b}=$ $\left(\tilde{h}, 0, \mathcal{V}_{2}\right)^{t r}$ and consider the following weak formulation of the stabilization problem (5):

$(\mathrm{wf})\left\{\begin{array}{l}\text { for all } \Psi \in V, \text { find } \tilde{U} \in V \text { such that } \\ \int_{\Omega} \Psi^{t r} \partial_{t} \tilde{U} d \Omega-\int_{\Omega}\left(\partial_{x} \Psi^{t r}\right) A \tilde{U} d \Omega \\ -\int_{\Omega}\left(\partial_{y} \Psi^{t r}\right) B \tilde{U} d \Omega \\ =-\int_{\Gamma_{n c}} \Psi^{t r} \tilde{U} \mathbf{u}_{n} d \sigma-\int_{\Gamma_{c x}} \Psi^{t r} A \mathcal{U}_{a} n_{x} d \sigma \\ -\int_{\Gamma_{c y}} \Psi^{t r} B \mathcal{U}_{b} n_{y} d \sigma .\end{array}\right.$

Let $\left\{e_{i} \in L^{2}(\Omega)^{3}, i \in \mathbb{N}\right\}$ be a hilbertian basis of $L^{2}(\Omega)^{3}$, we denote by $S^{k}$ the finite dimensional spaces defined as $S^{k}=\operatorname{vect}\left\{e_{i} 1 \leq i \leq k\right\}$. Since the initial condition $\tilde{U}^{0} \in$ $L^{2}(\Omega)^{3}$, there exists a subsequence $\left(\tilde{U}_{k}^{0}\right)_{k \geq 0} \subset S^{k}$ such that

$\tilde{U}_{k}^{0} \longrightarrow \tilde{U}^{0}$ in $L^{2}(\Omega)^{3}$ as $k \rightarrow+\infty$.

For any $k \geq 0$, we denote by $V_{k}$ the finite dimensional subspace of $V$ defined as

$V_{k}=\left\{\begin{array}{l}(\tilde{h}, \tilde{u}, \tilde{v}) \in L^{\infty}\left(0, T ; S^{k}\right): \text { for } \quad i=1,2,3 \\ X_{i}(\tilde{h}, \tilde{u}, \tilde{v}) \in H(\operatorname{Div}, Q), \\ (\tilde{u}, \tilde{v})_{\mid \Gamma_{n c}} \cdot \mathbf{n}=0, \quad \tilde{v}_{\mid \Gamma_{c x}}=0 \quad \text { and } \quad \tilde{u}_{\mid \Gamma_{c y}}=0\end{array}\right\}$ and we consider the following weak formulation

$$
\text { (wf-k) }\left\{\begin{array}{c}
\text { For all } \Psi \in V_{k}, \text { find } \tilde{U}_{k} \in V_{k} \text { such that } \\
\int_{\Omega} \Psi^{t r} \partial_{t} \tilde{U}_{k} d \Omega-\int_{\Omega}\left(\partial_{x} \Psi^{t r}\right) A \tilde{U}_{k} d \Omega \\
-\int_{\Omega}\left(\partial_{y} \Psi^{t r}\right) B \tilde{U}_{k} d \Omega \\
=-\int_{\Gamma_{n c}} \Psi^{t r} \tilde{U}_{k} \mathbf{u}_{n} d \sigma \int_{\Gamma_{c x}} \Psi^{t r} A \mathcal{U}_{a} n_{x} d \sigma \\
-\int_{\Gamma_{c y}} \Psi^{t r} B \mathcal{U}_{b} n_{y} d \sigma,
\end{array}\right.
$$

with $\tilde{U}_{k}^{0}$ as initial condition. We replace $\Psi$ by $\tilde{U}_{k}^{t r} D^{2}$ in (wfk) and we obtain the following estimation

$$
\begin{aligned}
\frac{d E_{k}(t)}{d t}= & -\left(\frac{\lambda_{1}^{a}}{\lambda_{3}^{a}}+\frac{\lambda_{2}^{a}}{\lambda_{3}^{a}} \alpha^{2}(t)\right) \int_{\Gamma_{c x}} \xi_{a_{1 k}}^{2} \bar{u} n_{x} d \sigma \\
& -\left(\frac{\lambda_{1}^{b}}{\lambda_{3}^{b}}+\frac{\lambda_{2}^{b}}{\lambda_{3}^{b}} \beta^{2}(t)\right) \int_{\Gamma_{c y}} \xi_{b_{1 k}}^{2} \bar{v} n_{y} d \sigma \\
& -\int_{\Gamma_{n c}} \hat{U}_{k}^{t r} \hat{U}_{k} \mathbf{u}_{n} d \sigma,
\end{aligned}
$$

where

$$
E_{k}(t)=\left\|\hat{U}_{k}\right\|_{L^{2}(\Omega)}^{2}=\int_{\Omega}\left(\tilde{h}_{k}^{2}+(\bar{h} / g) \tilde{u}_{k}^{2}+(\bar{h} / g) \tilde{v}_{k}^{2}\right) d \Omega .
$$

For $T>0$, we use the control laws (23)-(24) and integrate (29) over the time interval $[0, T]$ to write

$$
\begin{aligned}
& E_{k}(T)+\int_{0}^{T} \int_{\Gamma_{n c}} \hat{U}_{k}^{t r} \hat{U}_{k} \mathbf{u}_{n} d \sigma d t \\
& +\int_{0}^{T}\left(\frac{\lambda_{1}^{a}}{\lambda_{3}^{a}}+\frac{\lambda_{2}^{a}}{\lambda_{3}^{a}} \alpha^{2}(t)\right) \int_{\Gamma_{c x}} \xi_{a_{1 k}}^{2} \bar{u} n_{x} d \sigma d t \\
& +\int_{0}^{T}\left(\frac{\lambda_{1}^{b}}{\lambda_{3}^{b}}+\frac{\lambda_{2}^{b}}{\lambda_{3}^{b}} \beta^{2}(t)\right) \int_{\Gamma_{c y}} \xi_{b_{1 k}}^{2} \bar{v} n_{y} d \sigma d t=E_{k}(0) .
\end{aligned}
$$

For $\alpha$ and $\beta$ satisfying (26), from the relation (30) we deduce that $\left\|\hat{U}_{k}\right\|_{L^{2}\left(0, T ; L^{2}(\Omega)\right)},\left\|\hat{U}_{k}\right\|_{L^{2}\left(0, T ; L^{2}\left(\Gamma_{n c}\right)\right)},\left\|\xi_{a_{1 k}}\right\|_{L}^{2}(0, T$; $\left.L^{2}\left(\Gamma_{c x}\right)\right)$ and $\left\|\xi_{b_{1 k}}\right\|_{L^{2}\left(0, T ; L^{2}\left(\Gamma_{c y}\right)\right)}$ are bounded. Since the function spaces $L^{2}\left(0, T ; L^{2}(\Omega)\right), L^{2}\left(0, T ; L^{2}\left(\Gamma_{n c}\right)\right), L^{2}(0, T$; $\left.L^{2}\left(\Gamma_{c x}\right)\right)$ and $L^{2}\left(0, T ; L^{2}\left(\Gamma_{c y}\right)\right)$ are reflexive Banach spaces for $T>0$, there exists a subsequence denoted again $\tilde{U}_{k}$ weakly converging to $\tilde{U}$. We integrate the weak form (wf-k) over the time interval $[0, T]$ to get 


$$
\begin{aligned}
& -\int_{0} \int_{\Omega}\left(\partial_{t} \Psi^{t r}\right) \tilde{U}_{k} d \Omega d t \\
& -\int_{\Omega} \Psi^{t r}(0, x, y) \tilde{U}_{k}(0, x, y) d \Omega \\
& +\int_{\Omega} \Psi^{t r}(T, x, y) \tilde{U}_{k}(T, x, y) d \Omega \\
& -\int_{0}^{T}\left(\partial_{\Omega} \Psi^{t r}\right) A \tilde{U}_{k} d \Omega d t-\int_{0}^{T}\left(\partial_{y} \Psi^{t r}\right) B \tilde{U}_{k} d \Omega d t \\
& =-\int_{0}^{T} \int_{\Gamma_{n c}}^{T} \Psi^{t r} \tilde{U}_{k} \mathbf{u}_{n} d \sigma d t-\int_{0}^{T} \int_{\Gamma_{c x}} \Psi^{t r} A \mathcal{U}_{a} n_{x} d \sigma d t \\
& -\int_{0}^{T} \int_{\Gamma_{c y}} \Psi^{t r} B \mathcal{U}_{b} n_{y} d \sigma d t .
\end{aligned}
$$

In order to pass to the limit, we choose $\Psi=\delta(t) \eta(x, y)$ where $\delta \in H^{1}(0, T)$ such that $\delta(T)=0$ and $\eta \in H^{1}(\Omega)$. By letting $k$ tend to $+\infty$ one obtains

$$
\begin{aligned}
& -\int_{0}^{T} \partial_{t} \delta(t) \int_{\Omega} \eta^{t r} \tilde{U} d \Omega d t-\delta(0) \int_{\Omega} \eta^{t r} \tilde{U}^{0}(., .) d \Omega \\
& -\int_{0}^{T} \delta(t) \int_{\Omega}\left(\partial_{x} \eta\right)^{t r} A \tilde{U} d \Omega d t-\int_{0}^{T} \delta(t) \int_{\Omega}\left(\partial_{y} \eta\right)^{t r} B \tilde{U} d \Omega d t \\
& =-\int_{0}^{T} \delta(t) \int_{\Gamma_{n c}} \eta^{t r} \tilde{U}_{n c} \mathbf{u}_{n} d \sigma d t-\int_{0}^{T} \delta(t) \int_{\Gamma_{c x}} \eta^{t r} A \mathcal{U}_{a} n_{x} d \sigma d t \\
& -\int_{0}^{T} \delta(t) \int_{\Gamma_{c y}} \eta^{t r} B \mathcal{U}_{b} n_{y} d \sigma d t .
\end{aligned}
$$

Let us consider now $(\delta, \eta) \in \mathcal{D}(0, T) \times \mathcal{D}(\Omega)$, then in the sense of distribution

$$
\partial_{t} \tilde{U}+A \partial_{x} \tilde{U}+B \partial_{y} \tilde{U}=0 .
$$

Multiplying once again Eq. (33) by $\Psi=\delta \eta$ it comes

$$
\begin{aligned}
& -\int_{0}^{T} \partial_{t} \delta(t) \int_{\Omega} \eta^{t r} \tilde{U} d \Omega d t \\
& -\delta(0)\left(\eta^{t r}, \tilde{U}(0, ., .)\right)_{H^{\frac{1}{2}}(\Omega), H^{-\frac{1}{2}}(\Omega)}^{T} \\
& -\int_{0}^{T} \delta(t) \int_{\Omega}\left(\partial_{x} \eta\right)^{t r} A \tilde{U} d \Omega d t-\int_{0} \delta(t) \int_{\Omega}\left(\partial_{y} \eta\right)^{t r} B \tilde{U} d \Omega d t \\
& =-\left(\delta(t) \eta,\left(A n_{x}+B n_{y}\right) \tilde{U}\right)_{H^{\frac{1}{2}}\left((0, T) \times \Gamma_{n c}\right), H^{-\frac{1}{2}}\left((0, T) \times \Gamma_{n c}\right)}
\end{aligned}
$$

$$
\begin{aligned}
& -n_{x}(\delta(t) \eta, A \tilde{U})_{H^{\frac{1}{2}}\left((0, T) \times \Gamma_{c x}\right), H^{-\frac{1}{2}}\left((0, T) \times \Gamma_{c x}\right)} \\
& -n_{y}(\delta(t) \eta, B \tilde{U})_{H^{\frac{1}{2}}\left((0, T) \times \Gamma_{c y}\right), H^{-\frac{1}{2}}\left((0, T) \times \Gamma_{c y}\right)} .
\end{aligned}
$$

By comparing (32) and (34) we identify the initial conditions $\tilde{U}(0, .,)=.\tilde{U}^{0}(.,$.$) , and the boundary conditions on the$ controlled portion $\Gamma_{c}$ :

on $\Gamma_{c x} \tilde{U}=\mathcal{U}_{a} \Longrightarrow(\tilde{u}, \tilde{v})=\left(\mathcal{V}_{1}, 0\right)$,

on $\Gamma_{c y} \quad \tilde{U}=\mathcal{U}_{b} \Longrightarrow(\tilde{u}, \tilde{v})=\left(0, \mathcal{V}_{2}\right)$.

It remain now to identify the boundary condition on the uncontrollable part $\Gamma_{n c}$. In the space $H^{\frac{1}{2}}\left((0, T) \times \Gamma_{n c}\right) \times$ $H^{-\frac{1}{2}}\left((0, T) \times \Gamma_{n c}\right)$, the equality

$\left(\delta(t) \eta,\left(A n_{x}+B n_{y}\right) \tilde{U}\right)=\left(\delta(t) \eta, \tilde{U} \mathbf{u}_{n}\right)$

holds if and only if $\tilde{u}_{x}+\tilde{v} n_{y}=0$ on $\Gamma_{n c}$. Then, the equality $\tilde{U}_{n c}=\tilde{U}_{\mid \Gamma_{n c}}$ is equivalent to $\tilde{u} n_{x}+\tilde{v} n_{y}=0$. The boundary condition on $\Gamma_{n c}$ of our control system (5) is then identified. As $E(t) \leq \liminf _{k \rightarrow \infty} E_{k}(t)$, the energy estimate (7) stems from (30) and the proof of the theorem is complete.

\section{3 effect of size $\alpha$ and $\beta$}

The optimality of the control is related to the energy decay rate: faster is the energy decay, better are the control laws. From the relations (27) and (28) it appears that the actuators $\alpha$ and $\beta$ are mapped to the control gains $\frac{\alpha-1}{\alpha+1}$ and $\frac{\beta-1}{\beta+1}$ respectively. In other terms, the control action is parametrized in terms of the size of $\alpha$ and $\beta$. It is then appropriate to analyze the size effect of actuators. Assuming that the steady velocity $\bar{u}$ and $\bar{v}$ are negative, we denote $\alpha^{*}$ and $\beta^{*}$ the optimal values of the control gains. The value $\alpha^{*}$ and $\beta^{*}$ correspond to the minimum values of $\alpha$ and $\beta$ from (26) which is obviously $\alpha^{*}=\beta^{*}=0$. That is matching to the non-reflecting boundary conditions on $\Gamma_{c}$ associated to the desired equilibrium $(\bar{h}, \bar{u}, \bar{v})$. It is worth noticing to precise this steady state since non-reflecting boundary conditions might stabilize the flow around another steady state. For $\alpha, \beta$ satisfying (26), the boundary velocities $\mathcal{V}_{1}$ and $\mathcal{V}_{2}$ vanish instantaneously if and only if the perturbation height is null at the controlled portion. However it stay null only if the perturbation state is null over all the domain $\Omega$. Otherwise the traveling waves from the interior of $\Omega$ will reach the controlled portion before the time-constant runs out. The control law will then upload non-null value. One of the most important aspects of a control law is the cost of its practical implementation. This proposed control is cost-effective since it requires measurement only of the local water level on the boundary portion $\Gamma_{c}$. 


\section{Numerical results}

In this section, we present numerical experiments and discuss properties of the control law. First, we perform numerical simulations for the linearized control problem (5) and observe the energy behavior according to the designed controllers $\mathcal{V}_{1}$ and $\mathcal{V}_{2}$. We apply after the designed controllers to the nonlinear SW equations to see how the proposed method works with a real flow regulation problem.

\subsection{2-D linearized shallow water control}

It aims here to present numerical simulations describing the energy $E(t)$ behavior provided by local boundary controllers $\mathcal{V}_{1}$ and $\mathcal{V}_{2}$ defined at (27)-(28). For that, we consider the linear stabilization problem (5) in a square domain $\Omega$. The objective in this test is to vanish the perturbation state $(\tilde{h}, \tilde{u}, \tilde{v})$.

Numerical upwind methods use propagating information along characteristics variables as waves with different speeds and directions given by speed's signs. Those method are appropriate for solving linear hyperbolic symmetric model. Thus, the first order upwind finite volumes method (see [11]) combined with Strang splitting is applied to the hyperbolic IBVP (5). The square domain $\Omega=[0,20] \times[0,20]$ is meshed with $40 \times 40$ cells. The steady state parameters are $\bar{h}=0.75 \mathrm{~m}$ and $\bar{u}=\bar{v}=0.25 \mathrm{~ms}^{-1}$. The controlled portions are given by $\Gamma_{c x}=\{x=0$ and $0 \leq y \leq 20\}$ and $\Gamma_{c y}=$ $\{0 \leq x \leq 20$ and $y=0\}$, the uncontrolled part is then $\Gamma_{n c}=$ $\{x=20$ and $0 \leq y \leq 20\} \cup\{0 \leq x \leq 20$ and $y=20\}$. We have plotted the energy $E(t)$ variation in several cases for the following initial perturbation conditions

$\tilde{h}^{0}(x, y)=1 m$ and $\left(\tilde{u}^{0}, \tilde{v}^{0}\right)(x, y)=(0,0)$.

The duration $T=16 s$ is chosen enough to observe waves traveling since time-constant, computed from the wave speed $c$ and the dimensions of the domain, is less than $10 s$. For $\alpha$ and $\beta$ belonging to $[-0.91,0.91]$, the inequalities (26) are satisfied and by way the energy $E(t)$ decay is guaranteed. It is apparent from the previous Fig. 2 that the boundary controllers $\mathcal{V}_{1}$ and $\mathcal{V}_{2}$ ensure decay of the perturbation energy $E(t)$. It is important to remark that the energy $E(t)$, after the initial increasing, decay is monotone as expected from the theoretical result. Inspection of the energy curves shows also that quicker perturbation energy decay are provided by actuators $\alpha$ and $\beta$ values neighboring 0 . Then, the non-reflecting boundary conditions $\left(\alpha=\beta=0\right.$ ) on $\Gamma_{c}$ turn to the limit case of quicker energy decay.

\subsection{Control of 2-D nonlinear shallow water equations}

In this subsection, we apply the designed controllers to the 2-D nonlinear SW equations in order to access the effectiveness of the proposed method. We deal with the conservative form of the 2-D nonlinear SW equations and we perform two types of numerical tests. The first test consists in describing controlled waves dynamic behavior in a square domain. The second test concerns in illustrating the perturbation energy decay on a square domain with respect to several values of the actuators $\alpha$ and $\beta$. The simulations are performed using high-order finite volumes method. We apply the controller $\mathcal{V}_{1}$ and $\mathcal{V}_{2}$ defined at (27) and (28) to the 2-D nonlinear conservative $\mathrm{SW}$ system

$$
\begin{aligned}
& \partial_{t} W+\partial_{x} \mathbf{f}(W)+\partial_{y} \mathbf{g}(W)=0 \text { in } \mathcal{Q}, \\
& W(0, x, y)=W^{0}(x, y) \text { in } \Omega, \\
& (h u, h v)=(\bar{h} \bar{u}, \bar{h} \bar{v})+\tilde{h}\left(\mathcal{V}_{1}, 0\right) \text { on } \Gamma_{c x}, \\
& (h u, h v)=(\bar{h} \bar{u}, \bar{h} \bar{v})+\tilde{h}\left(0, \mathcal{V}_{2}\right) \text { on } \Gamma_{c y}, \\
& (h u, h v) \cdot \mathbf{n}=h(\bar{u}, \bar{v}) \cdot \mathbf{n} \text { on } \Gamma_{n c},
\end{aligned}
$$

Fig. 2 Energy variation in time computed from the linearized control system (5) for different values of $\alpha$ and $\beta$

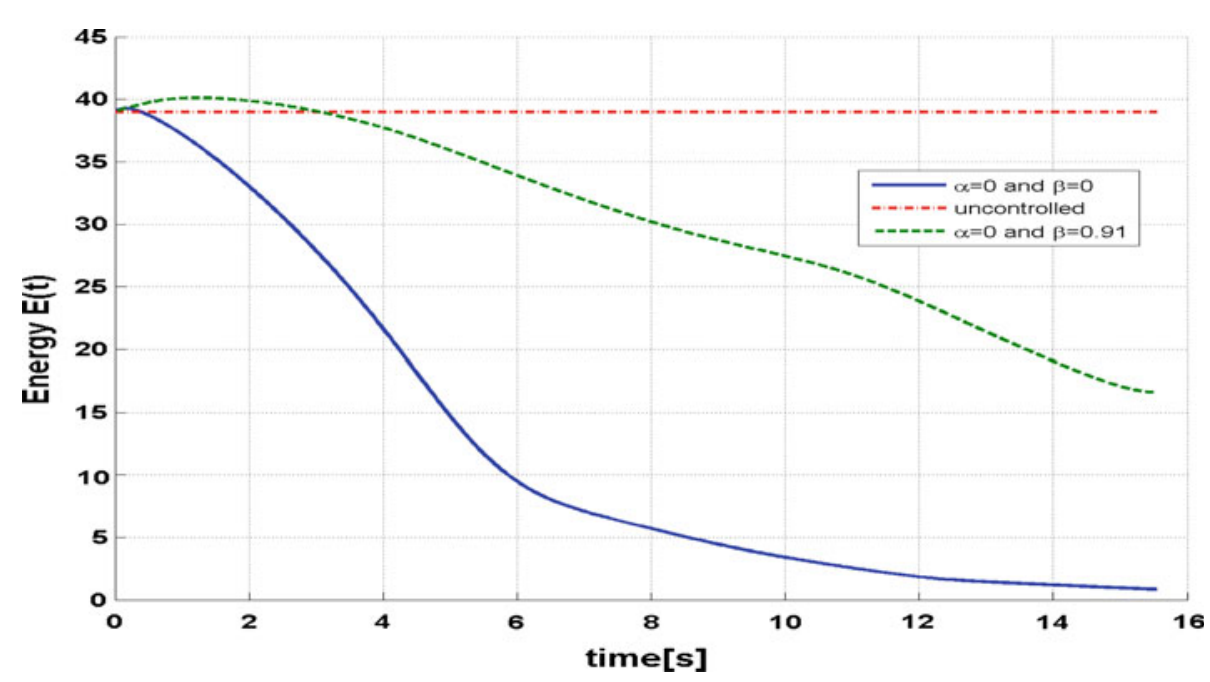


where

$W=\left[\begin{array}{l}h \\ h u \\ h v\end{array}\right], \mathbf{f}=\left[\begin{array}{l}h u \\ h u^{2}+\frac{1}{2} g h^{2} \\ h u v\end{array}\right], \mathbf{g}=\left[\begin{array}{l}h v \\ h u v \\ h v^{2}+\frac{1}{2} g h^{2}\end{array}\right]$.

The general form of the high order finite volume wavepropagation algorithm for the 2-D nonlinear SW system is:

$$
\begin{aligned}
Q_{i j}^{n+1}= & Q_{i j}-\frac{\Delta t}{\Delta x}\left(\mathcal{A}^{+} \Delta Q_{i-\frac{1}{2} j}+\mathcal{A}^{-} \Delta Q_{i+\frac{1}{2} j}\right) \\
& -\frac{\Delta t}{\Delta y}\left(\mathcal{B}^{+} \Delta Q_{i j-\frac{1}{2}}+\mathcal{B}^{-} \Delta Q_{i j+\frac{1}{2}}\right) \\
& -\frac{\Delta t}{\Delta x}\left(\tilde{F}_{i+\frac{1}{2} j}-\tilde{F}_{i-\frac{1}{2} j}\right) \\
& -\frac{\Delta t}{\Delta y}\left(\tilde{G}_{i j+\frac{1}{2}}-\tilde{G}_{i j-\frac{1}{2}}\right) .
\end{aligned}
$$
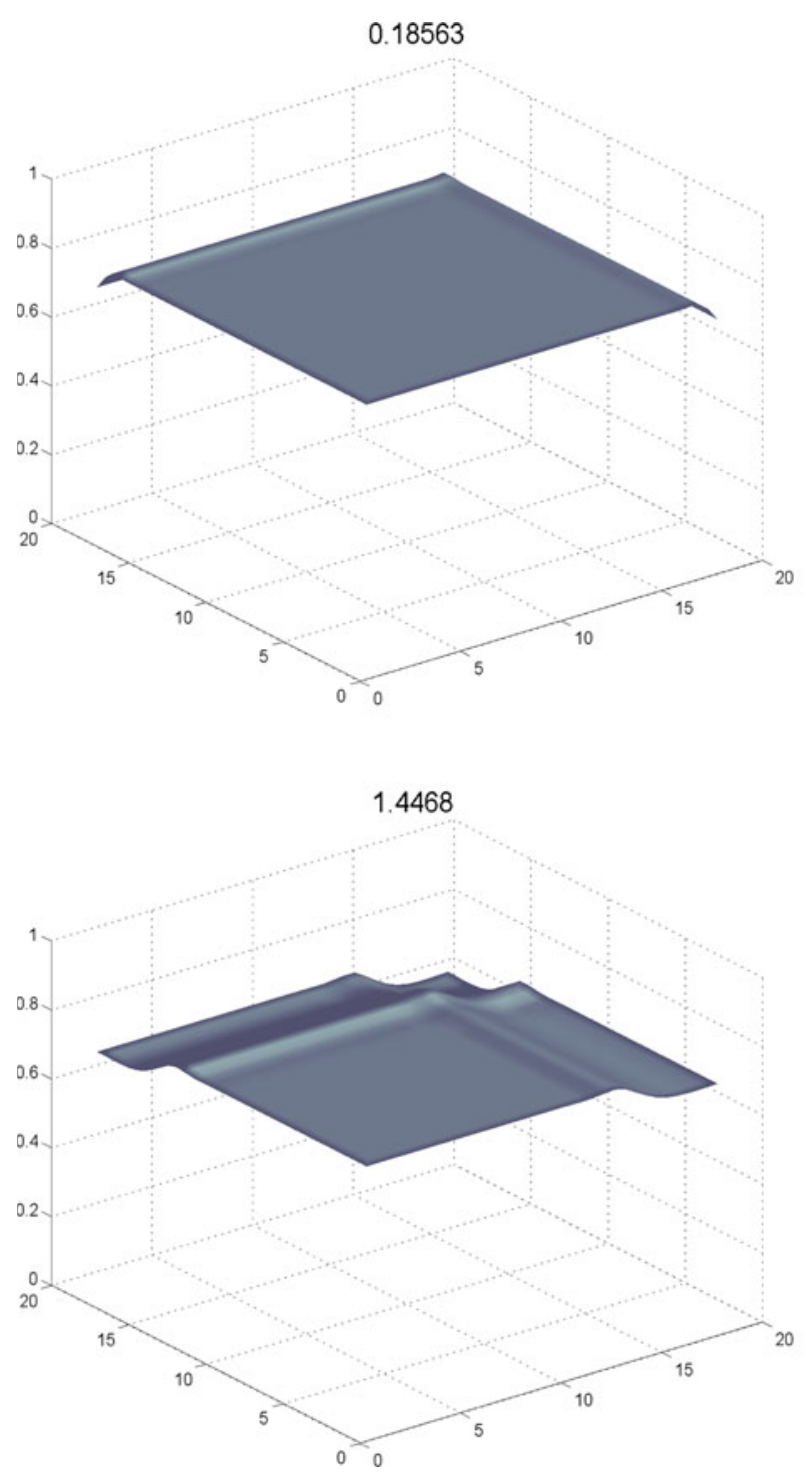

The quantity $Q_{i j}$ is the approximation to the cell average of the exact solution $W$

$Q_{i j} \approx \frac{1}{\Delta x \Delta y} \iint_{C_{i j}} W\left(t^{n}, x, y\right) d x d y$.

The two dimensional grid cell $C_{i j}$ is defined as $C_{i j}=$ $\left[x_{i-\frac{1}{2}}, x_{i+\frac{1}{2}}\right] \times\left[y_{j-\frac{1}{2}}, y_{j+\frac{1}{2}}\right]$, where $x_{i+\frac{1}{2}}-x_{i-\frac{1}{2}}=\Delta x$ and $y_{j+\frac{1}{2}}-y_{j-\frac{1}{2}}=\Delta y$. The time $t^{n}=t^{n-1}+\Delta t$, the time step $\Delta t$ is non-constant and is computed under the CFL stability condition. The fluctuations $\mathcal{A}^{ \pm} \Delta Q$ and $\mathcal{B}^{ \pm} \Delta Q$ represent the first order update to the cell value $C_{i j}$ resulting from the Riemann problems at the edges. Second order correction terms $\tilde{F}_{i \pm \frac{1}{2} j}$ and $\tilde{G}_{i j \pm \frac{1}{2}}$ are incorporated as in 1-D space, based on the waves obtained from the 1-D Riemann solution normal to
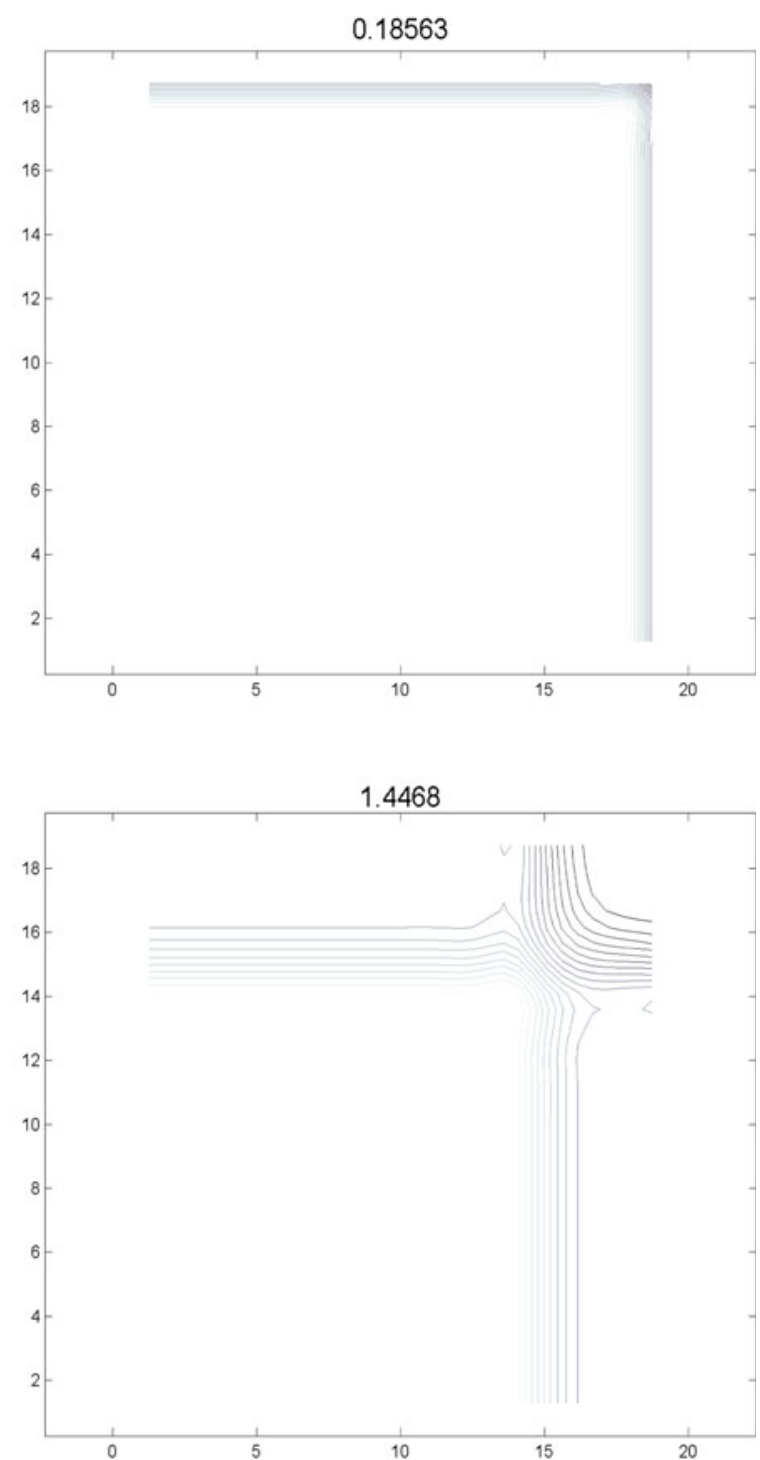

Fig. 3 3-D view (left) and 2-D contours view (right) of controlled waves dynamic, form top to bottom: water level $h$ at times $t=0.18563$, $t=$ $1.4468, t=3.6068, t=4.9749$ and $t=7.7041$ 

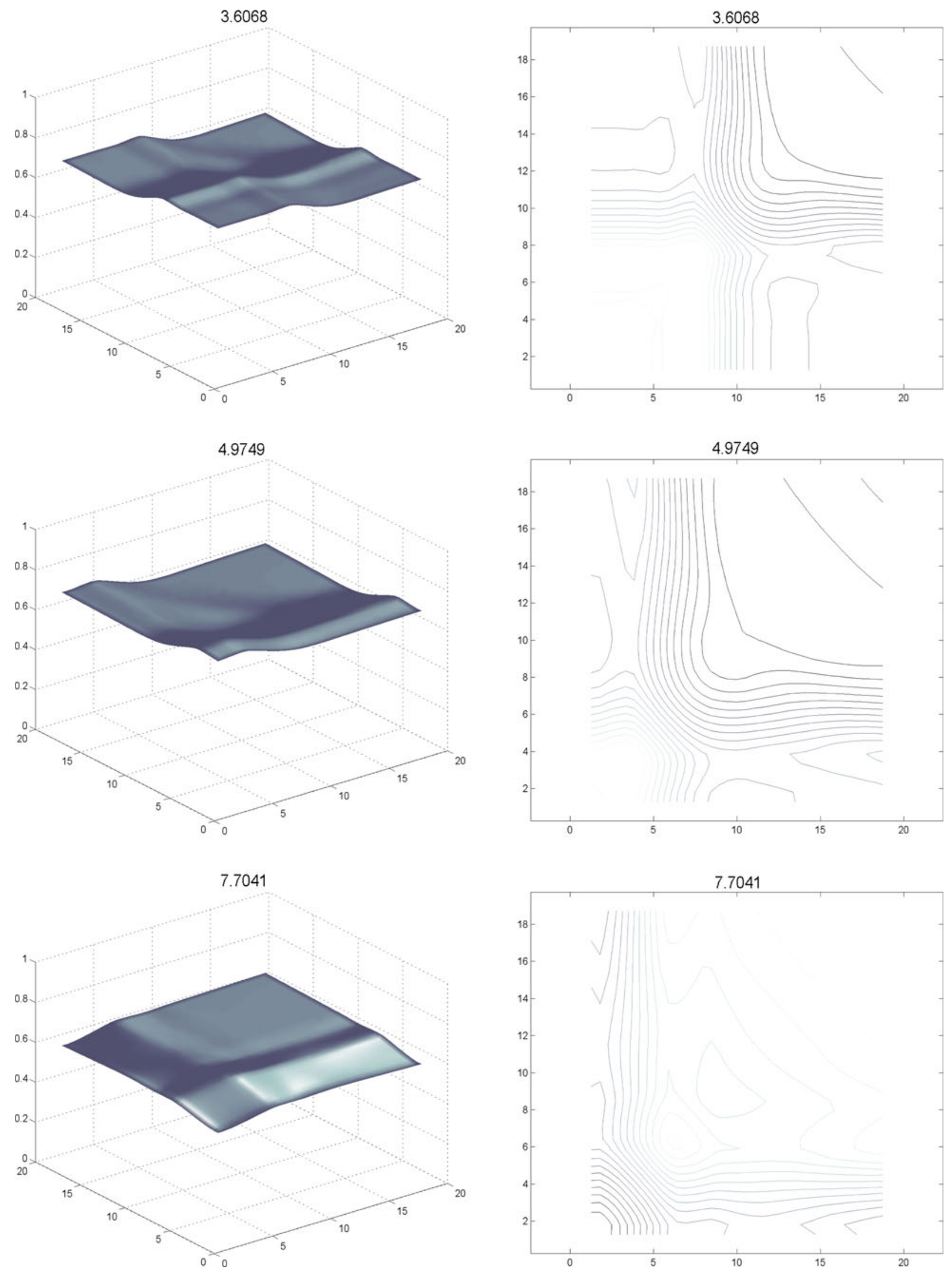

Fig. 3 continued 
Fig. 4 Residual energy decay comparison with respect to $\alpha$ and $\beta$

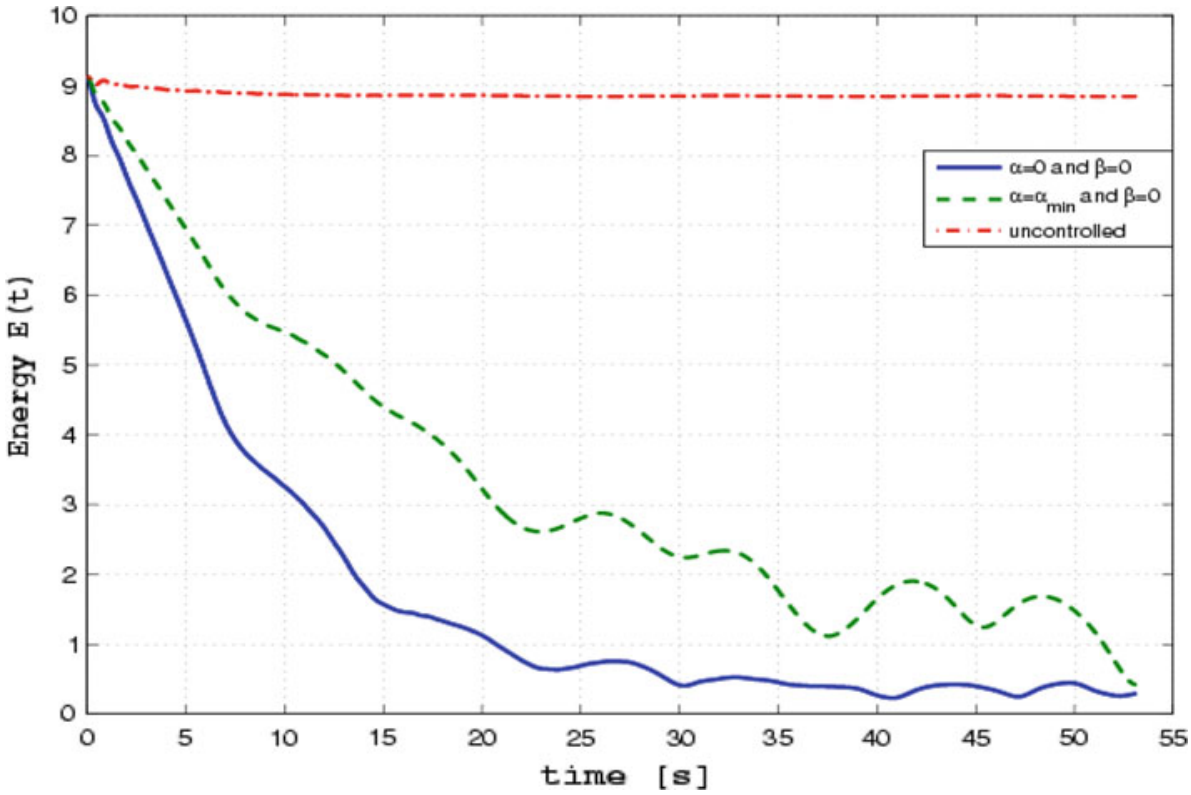

each edge. We have limited the flux corrections $\tilde{F}_{i-\frac{1}{2} j}$, etc., by the minmod limiter (see $[10,11]$ ).

We have considered a rectangular domain $R_{\Omega}$ which contains our computational domain $\Omega$. We have used structured grids to discretize the 2-D domain $R_{\Omega}$. Between two consecutive time steps, the flow vector is set to zero (i.e $(h u, h v)=(0,0))$ for the cells localized strictly outside the domain $\Omega$. Respect to the boundary conditions, we have dealt with $(h u, h v)=(\bar{h} \bar{u}, \bar{h} \bar{v})+\tilde{h}\left(\mathcal{V}_{1}, 0\right)$ over the cells crossing $\Gamma_{c x}$ and $(h u, h v)=(\bar{h} \bar{u}, \bar{h} \bar{v})+\tilde{h}\left(0, \mathcal{V}_{2}\right)$ over the cells crossing $\Gamma_{c y}$.

\subsubsection{Test 1: Controlled 2-D waves dynamic}

We run the previous high order finite volumes algorithm (38) in a square domain $\Omega=[0,20] \times[0,20]$ with the following initial conditions

$h^{0}(x, y)=0.75 \mathrm{~m} \quad$ and $\quad\left(u^{0}, v^{0}\right)(x, y)=(0,0)$ in $\Omega$.

We brought the state (40) to the water level $\bar{h}=0.50 \mathrm{~m}$ with the constant velocity vector $(\bar{u}, \bar{v})=-(0.25,0.25)$ under the stability condition $C F L=0.2$. The grid cell dimensions are $\Delta x=\Delta y=1$. One considers actuators values $\alpha=0$ and $\beta=0$. This choice makes easy the observations of the moving waves.

The computed free surface elevation is shown at several times in the previous figure (Fig. 3). The feedback boundary control vector $\left(\mathcal{V}_{1}, 0\right)$ defined on $\Gamma_{c x}=\{x=20, \quad y \in[0,20]\}$ and $\left(0, \mathcal{V}_{2}\right)$ defined on $\Gamma_{c y}=\{x \in[0,20] \quad y=20\}$ generate incoming waves with low magnitude. Those waves travel as unidirectional to the boundary $\Gamma_{n c}$ where they will be reflected. Those reflected waves and the incoming ones from $\Gamma_{c}$ give rise shock waves moving to $\Gamma_{c}$. The setting boundary conditions on $\Gamma_{c}$ inhibit the reflection of those waves and by the way provide energy lost. The generated shock waves becomes more conspicuous on a strict rectangular domain.

\subsubsection{Test 2: Actuators effect for the nonlinear problem}

We study the actuators effect in the decay of the energy of the perturbation state. The simulation are performed on the square $\Omega=[0,20] \times[0,20]$. The grid dimensions are $\Delta x=$ $\Delta y=0.5 \mathrm{~m}$ and the scheme stability condition is $C F L=$ 0.2 . One has considered perturbed initial height

$h^{0}(x, y)=0.75+0.125 \sin (x) \sin (y)$ and $\left(u^{0}, v^{0}\right)=(0,0)$.

This experiment consists in reducing the water level to $\bar{h}=0.5 m$ with the constant velocity vector $(\bar{u}, \bar{v})=$ $(-0.25,-0.25)$. We denote by $\mathbb{E}$ the residual energy computed form the nonlinear controlled system :

$\mathbb{E}(t)=\int_{\Omega}\left((h-\bar{h})^{2}+\frac{\bar{h}}{g}(u-\bar{u})^{2}+\frac{\bar{h}}{g}(v-\bar{v})^{2}\right) d \Omega$.

We have computed the energy $\mathbb{E}(t)$ for several values of $\alpha$ and $\beta$.

The previous figure (Fig. 4) compares residual energy decays with respect to the actuators. Since we dealt with perturbed initial conditions, the uncontrolled residual energy ( $\alpha=\beta=1)$ becomes constant after a while. The result shows that the energy decays faster for non-reflecting boundary conditions $(\alpha=0$ and $\beta=0$ ). The plotted energy for $\alpha=\alpha_{\text {min }}= \pm \sqrt{-\frac{\lambda_{2}^{a}}{\lambda_{1}^{a}}}$ and $\beta=0$ is an intermediate 
case between the non-reflecting boundary conditions and the minimal cost of control effort. The observed non-monotone decrease of the residual energy might be interpreted as consequence of the linearization. In fact, this last transformation imposes the approximation $E(t) \approx \mathbb{E}(t)$.

\section{Conclusion}

In this paper a sufficient stability condition for velocities and water level in 2-D channel flow has been described and analyzed. We have addressed the problem by local boundary feedback control for the 2-D SW equations. The control law is designed for a linear stabilization problem by acting on the incoming characteristic variables. Those lasts in 1-D view had been wrote out means symmetrization of the flux matrices. First order upwind finite volumes method is implemented with Strang splitting to describe perturbation energy (computed from the linearized model) monotone decrease. Moreover, numerical experiments with a second order accurate algorithm, Roe linearization shows that the designed boundary controllers work on the 2-D nonlinear SW equations. In future, we aim to investigate improvements to the asymptotic stability by seeking how to build dissipative boundary conditions using characteristic variables for the 2$\mathrm{D}$ channel flow as it is done for the 1-D case. We shall address also the exponential stability of the 2-D SW equations using the same approach.

\section{References}

1. Aamo OM, Krstic M, Bewley TR (2003) Control of mixing by boundary feedback in 2-d channel flow. Automatica 39:1597-1606
2. Balogh A, Liu WJ, Krstic M (2001) Stability enhancement by boundary control in 2-d channel flow. IEEE Transact Autom Control 46(11)

3. Coron J-M, d'Andréa-Novel B, Bastion G (2007) A strict Lyapunov function for boundary control of hyperbolic systems of conservation laws. IEEE Transact Autom Control 52(1):2-11

4. d'Andréa-Novel B, Coron J-M, Bastin G (1999) A Lyapunov approach to control irrigation canals modeled by saint venant equations. In: CD-Rom Proceedings European Control Conference ECC99, Paper F1008-5

5. de Halleux J, Prieur C, Coron J-M, d'Andréa-Novel B, Bastin G (2003) Boundary feedback control in networks of open-channels. Automatica 39:1365-1376

6. Dia BM, Sene A, Oppelstrup J (2011) Exponential stability of 1-D hyperbolic system for controlling 2-D hydraulic flow. (in review)

7. Diagne A, Bastin G, Coron J-M (2011) Lyapunov exponential stability of 1-d linear hyperbolic systems of balance laws. Automatica

8. Girault V, Riviart PA (1986) Finite elements methods for navier stokes equations. Theory and algorithms, volume 136 of mathematical surveys and monographs. Springer, Providence

9. Lefevre L, Chen ML, Georges D (2002) Infinite dimensional LQ control of an open channel hydraulic system. Fourth Asian control conference

10. Leveque RJ (1997) Wave propagation algorithms for multidimensional hyperbolic systems. J comput Phys 327-253

11. Leveque RJ (2002) Finite volumes methods for hyperbolic problems. Cambridge Texts in Applied Mathematics, Cambridge

12. Lions JL (1968) Controle optimale des systémes gouvernés par des quations aux drives partielles. Dunod et Gauthier-Villars, Paris

13. Russell DL (1978) Controllability and stabilizability theory for linear partial differential equations: recent progress and open questions. Soc Ind Appl Math, vol 20

14. Rodriguez LS, Batlle V, Perez RR (2007) Fractional robust control of main irrigation canals with variable dynamic parameters. Control Eng pract 14:673-686 